\title{
Price-Taker Offering Strategy in Electricity Pay-as-Bid Markets
}

\author{
Mazzi, Nicolò; Kazempour, Jalal; Pinson, Pierre
}

Published in:

IEEE Transactions on Power Systems

Link to article, DOI:

10.1109/TPWRS.2017.2737322

Publication date:

2018

Document Version

Peer reviewed version

Link back to DTU Orbit

Citation (APA):

Mazzi, N., Kazempour, J., \& Pinson, P. (2018). Price-Taker Offering Strategy in Electricity Pay-as-Bid Markets. IEEE Transactions on Power Systems, 33(2), 2175 - 2183. https://doi.org/10.1109/TPWRS.2017.2737322

\section{General rights}

Copyright and moral rights for the publications made accessible in the public portal are retained by the authors and/or other copyright owners and it is a condition of accessing publications that users recognise and abide by the legal requirements associated with these rights.

- Users may download and print one copy of any publication from the public portal for the purpose of private study or research.

- You may not further distribute the material or use it for any profit-making activity or commercial gain

- You may freely distribute the URL identifying the publication in the public portal

If you believe that this document breaches copyright please contact us providing details, and we will remove access to the work immediately and investigate your claim. 


\title{
Price-Taker Offering Strategy in Electricity Pay-as-Bid Markets
}

\author{
Nicolò Mazzi, Jalal Kazempour, Member, IEEE, and Pierre Pinson, Senior Member, IEEE
}

\begin{abstract}
The recent increase in the deployment of renewable energy sources may affect the offering strategy of conventional producers, mainly in the balancing market. The topics of optimal offering strategy and self-scheduling of thermal units have been extensively addressed in the literature. The feasible operating region of such units can be modeled using a mixed-integer linear programming approach, and the trading problem as a linear programming problem. However, the existing models mostly assume a uniform pricing scheme in all market stages, while several European balancing markets (e.g., in Germany and Italy) are settled under a pay-as-bid pricing scheme. The existing tools for solving the trading problem in pay-as-bid electricity markets rely on non-linear optimization models, which, combined with the unit commitment constraints, result in a mixed-integer non-linear programming problem. In contrast, we provide a linear formulation for that trading problem. Then, we extend the proposed approach by formulating a two-stage stochastic problem for optimal offering in a two-settlement electricity market with a pay-as-bid pricing scheme at the balancing stage. The resulting model is mixed-integer and linear. The proposed model is tested on a realistic case study against a sequential offering approach, showing the capability of increasing profits in expectation.
\end{abstract}

Index Terms-Pay-as-bid, offering strategy, stochastic programming, mixed-integer linear program, thermal unit.

\section{NOMENCLATURE}

\section{Indices and Sets}

$\begin{array}{ll}i, i^{\prime}(I) & \text { Indices of day-ahead market price scenarios } \\ j, j^{\prime}(J) & \text { Indices of balancing market price scenarios } \\ s(S) & \text { Index of generation blocks } \\ k(K) & \text { Index of time intervals } \\ \Pi & \text { Feasible region of the unit's offer curves } \\ \Omega & \text { Feasible region of the unit's operation }\end{array}$

\section{Parameters}

$\begin{array}{ll}\lambda_{i k}^{\mathrm{DA}} & \text { Day-ahead market price [€/MWh] } \\ \lambda_{i j k}^{\mathrm{BA}} & \text { Balancing market price [€/MWh] } \\ M_{j j^{\prime} k}^{\mathrm{UP}} & \text { Acceptance matrix for up-regulation offers } \\ M_{j j^{\prime} k}^{\mathrm{DW}} & \text { Acceptance matrix for down-regulation offers } \\ \bar{E} & \text { Capacity of the unit [MW] } \\ \underline{E} & \text { Minimum power limit of the unit [MW] } \\ R^{\mathrm{UP}}, R^{\mathrm{DW}} & \text { Ramp-up and down limits [MW/h] }\end{array}$

N. Mazzi is with the Department of Industrial Engineering, University of Padova, Padova, Italy (e-mail: nicolo.mazzi@ dii.unipd.it).

J. Kazempour and P. Pinson are with the Department of Electrical Engineering, Technical University of Denmark, Kgs. Lyngby, Denmark (e-mail: seykaz@elektro.dtu.dk; ppin@elektro.dtu.dk). J. Kazempour and P. Pinson are partly supported by the Danish Innovation Fund through the projects 5s - Future Electricity Markets (12-132636/DSF) and CITIES (DSF-130500027B).
$E_{s} \quad$ Size of generation block $s[\mathrm{MW}]$
$C^{\mathrm{UP}}, C^{\mathrm{DW}}$ Start-up and shut-down costs [€]
$C_{0} \quad$ Cost at the minimum production level [€]
$C_{s} \quad$ Marginal cost of generation block $s$ [€/MWh]
$\pi_{i}^{\text {DA }} \quad$ Probability of day-ahead price scenario $i$
$\pi_{i j}^{\mathrm{BA}} \quad$ Probability of balancing price scenario $j$, pro- vided that day-ahead price scenario $i$ realizes

Variables

$q_{i k}^{\mathrm{DA}} \quad$ Quantity offer at day-ahead market [MWh]

$o_{i j k}^{\mathrm{UP}}, o_{i j k}^{\mathrm{DW}} \quad \mathrm{Up} /$ down regulation incremental offer [MWh]

$q_{i j k}^{\mathrm{UP}}, q_{i j k}^{\mathrm{DW}} \quad \mathrm{Up} /$ down regulation quantity offer [MWh]

$q_{i j k}^{\mathrm{A}} \quad$ Total production quantity [MWh]

$c_{i j k} \quad$ Operational cost [€/MWh]

$\rho_{i j k}^{\mathrm{UP}}, \rho_{i j k}^{\mathrm{DW}} \quad \mathrm{Up} /$ down regulation profit $[€]$

$y_{i j k}, z_{i j k} \quad$ Start-up/shut-down (binary) status of the unit

$u_{i j k} \quad$ Commitment (binary) status of the unit

\section{Functions \\ $g(\cdot), h(\cdot) \quad$ Operational cost functions}

\section{INTRODUCTION}

$\mathbf{I}$ $\mathrm{N}$ recent years the power sector has experienced a significant increase in the deployment of renewable energy sources, such as wind and solar power. These sources are usually traded at zero marginal cost and their growing penetration is leading to a decrease of the prices in the day-ahead market [1]-[2]. Moreover, they can only be predicted with a limited accuracy, thus leading to real-time imbalances and increasing the need for balancing energy. These changes may affect the strategy of the conventional producers in both day-ahead and balancing markets.

\section{A. Literature Review}

The optimal offering strategy and self-scheduling of conventional thermal units have already been widely studied in the literature. Ref. [3] addresses the optimal response of a thermal generator to a given set of electricity market prices in terms of both energy and reserve. A mixed-integer linear programming (MILP) problem is developed considering a nonconvex cost function, as well as its start-up costs, ramp rates and minimum-up and -down constraints. The same authors in [4] propose a detailed formulation to model start-up and shut-down characteristics of a thermal generator. Other works, such as [5] and [6], include risk measures while optimizing 
the self-scheduling problem of thermal units. References [3][6] demonstrate that a detailed modeling of the generator's feasibility region and its production cost function may be essential for deriving its optimal self-scheduling. Indeed, the inter-temporal constraints (e.g., ramping constraints) and nonconvex costs (e.g., start-up and shut-down costs) may affect the optimal solution. In this context, the pioneering paper [7] presents an offering strategy for a price-taker producer under price uncertainty. It develops a set of rules that aim to translate the results of a self-scheduling problem into market offers. Ref. [8] presents an algorithm for offering and selfscheduling of a unit including risk management. Ref. [9] presents an offering strategy for a price-taker power producer that aims to maximize profit expectation while hedging against possible infeasible schedules. Other works relax the price-taker assumption and develop tools for strategic offering considering the impact of power producer's decisions on market prices. This can be done through a residual demand model [10] or a bilevel optimization setup [11]-[12].

By analyzing the optimization models in [3]-[12], we can identify two different sets of variables and constraints. The first set defines the feasibility region and the cost function of the production unit. For instance, references [3]-[6] show how to successfully model it as a MILP problem. The second set simulates the trading problem, i.e., how the power producer participates in the market (e.g., through non-decreasing stepwise offering curves), while considering the market clearing mechanism (endogenously or exogenously) and the pricing scheme (e.g., uniform or pay-as-bid). The trading problem can be modeled using a linear programming (LP) approach [13], under price-taker assumptions and uniform pricing scheme. However, even though European day-ahead electricity markets are mostly settled under a uniform pricing scheme, several balancing markets (e.g., in Germany and Italy [14]) are settled under a pay-as-bid pricing scheme.

The topic of trading under a pay-as-bid scheme and price uncertainty has not been extensively addressed in the literature. An analysis on optimal offering under pay-as-bid and uniform pricing schemes is presented in [15] and [16]. They obtain profit expectation and variance for both pricing schemes, while assuming that the market price follows a uniform distribution. Ref. [17] proposes a methodology that aims to maximize the profit expectation in a day-ahead pay-as-bid auction for power system reserve. Offering strategies for a joint energy and spinning reserve market under pay-as-bid pricing are presented in [18] and [19]. Risk aversion is introduced in [20]. References [15]-[20] show how to model the trading problem under payas-bid pricing scheme using a non-linear programming (NLP) approach. However, they do not consider an accurate modeling of production unit's operational constraints. Introducing the feasibility region would result in a mixed-integer non-linear problem (MINLP), which may have high computational cost and, generally, do not guarantee the optimality of the solution. It is worth mentioning that [19] proposes to solve the trading problem in pay-as-bid markets under price uncertainty with a two-step approach, obtaining the expected profit as a linear function of the quantity offer. However, this approach is not applicable in case of problems with inter-temporal constraints or with more complex cost functions.

\section{B. Approach and Contributions}

Compared to the available literature, this paper provides a novel approach that allows to cast the optimal price-taker trading problem in pay-as-bid markets under price uncertainty as an LP problem. For that purpose, continuous random variables (i.e., market-clearing prices) are represented as discrete variables. We test our formulation against the existing continuous NLP alternative. In a simple setup, these two models bring similar optimal solutions, while our LP approach drastically reduces the computational cost. Hence, we demonstrate that our LP model is a good approximation of the NLP one. However, the value of our LP formulation, with respect to the NLP one, arises when including the feasible operating region of the unit.

Then, we use the proposed LP approach to build a multistage stochastic programming problem with recourse. This efficient decision-making tool could be used by a price-taker conventional producer to derive its best day-ahead market offer curves. In line with current practice in several European electricity markets, we consider a two-settlement market framework, in which the day-ahead market is cleared based on a uniform pricing scheme, while a pay-as-bid pricing scheme is used in the balancing stage. The forecast market prices in both stages are given but uncertain. This uncertainty is properly characterized by generating a set of foreseen scenarios. The resulting model is a stochastic MILP problem, where non-convexities (i.e., binary variables) arise from the unit commitment constraints. To the best of our knowledge, this kind of stochastic MILP optimization model for obtaining the offering strategy of a price-taker thermal producer in a two-settlement electricity market with a pay-as-bid pricing scheme in the balancing stage is not available in the literature. It is worth mentioning that [12] provides a formulation for obtaining optimal offering curves in markets settled under a pay-as-bid pricing scheme for a price-maker producer. However, market problems with equilibrium constraints may have high computational cost and rely on strong assumptions on opponents' behavior. Hence, when the production unit has a negligible impact on the market, a price-maker setup may not be the preferable choice.

\section{Paper Organization}

The remaining of the paper is organized as follows. Section II presents the electricity market framework, the modeling assumptions, and the methodology for generating market price scenarios. Section III provides an overview of the existing NLP setup as well as the proposed LP setup for deriving the offering strategy of a price-taker producer under a pay-as-bid pricing scheme. Then, Section IV extends the proposed approach and develops a two-stage model for a price-taker producer to derive its best offering curves in the day-ahead market, considering a pay-as-bid pricing scheme in the balancing stage. Section $\mathrm{V}$ presents a verification test to assess the performance of the proposed LP trading model, as well as an application of the two-stage model using a realistic test case. Finally, the conclusions are drawn in Section VI. 


\section{Modeling Assumptions And Market Price SCENARIOS}

We consider a single conventional producer that trades in a two-settlement electricity market framework. The day-ahead market is cleared once a day, at noon, simultaneously for the whole 24 hourly trading periods of the following day. Generators are remunerated under a uniform pricing scheme in the day-ahead market. Then, a balancing market is cleared separately per each hourly interval, one hour prior to real-time operation. The provision of balancing energy is remunerated under a pay-as-bid pricing scheme. The intra-day trading floor is neglected for the sake of simplicity.

The power producer is assumed to be price-taker in both day-ahead and balancing markets. Hence, the market prices within the offering strategy problem of that producer are exogenous, but still uncertain. We model those uncertainties using a set of scenarios. Uncertainty characterization is a critical input to stochastic optimization. The quality of the solution of a stochastic optimization model is indeed strongly influenced by the quality of the scenarios provided as input. Given that the purpose of this paper is to analyze and test an optimization model, we exploit a fundamental model for generating market prices, instead of using real market data. This fundamental model generates a set of electricity market price forecasts, which is required as an input to our proposed offering strategy.

In the fundamental market model we assume, for the sake of simplicity, that the only stochastic generation is wind power generation. A dataset of wind power forecasts for a wind farm located in Denmark is used. The wind power forecasts are re-scaled and assumed representative of the aggregated wind power production in the market area. At the day-ahead stage, we assume that the demand curve is linear, known, and different per each hourly interval. Conversely, the supply curve of conventional producers is quadratic and uncertain. To model this uncertainty, we consider the coefficient of the second-degree term (i.e., $\gamma_{k}$, where $k$ is the index of time interval) as a random variable with known marginal distribution. The methodology for fitting such distribution is beyond the scope of the paper. The coefficient of the firstdegree term is also considered known to simplify the process of scenario generation. Then, we assume that the stochastic generation is offered in the day-ahead market at its mean forecast and at zero price.

At the balancing stage, the supply curve is assumed known but different from the day-ahead one. Indeed, the participants in the balancing market (under pay-as-bid pricing scheme) do not offer their marginal cost, since they have to internalize the expected revenues into their market offers [15]. Therefore, we fix a negative price floor $\lambda_{0}$ and impose $\gamma_{k}^{\mathrm{BA}}=\eta \gamma_{k}$ $(\eta>1)$, where $\gamma_{k}^{\mathrm{BA}}$ is referred to the supply curve in the balancing market. Several factors may cause the real-time power imbalance in the system, e.g., errors in load and wind forecasts. For the sake of simplicity, we consider the wind stochasticity as the only source of uncertainty at the balancing stage. This simplifies the scenario generation process.

\section{A. Market Model}

The demand curve of the day-ahead market at hourly interval $k$ is

$$
p_{k}^{\mathrm{DA}, \mathrm{d}}=\alpha_{k}+\delta e_{k}^{\mathrm{DA}, \mathrm{d}},
$$

where $e_{k}^{\mathrm{DA}, \mathrm{d}}$ is the amount of energy demand at price $p_{k}^{\mathrm{DA}, \mathrm{d}}$. The parameters $\alpha_{k}$ and $\delta$ control the shape of the demand curve. For the same interval $k$, the supply curve is

$$
p_{k}^{\mathrm{DA}, s}= \begin{cases}0, & \text { if } \Delta_{k}^{\mathrm{DA}, \mathrm{s}} \leq 0 \\ \beta \Delta_{k}^{\mathrm{DA}, s}+\gamma_{k}\left(\Delta_{k}^{\mathrm{DA}, s}\right)^{2}, & \text { otherwise }\end{cases}
$$

where $\Delta_{k}^{\mathrm{DA}, s}=e_{k}^{\mathrm{DA}, s}-W_{k}^{\mathrm{DA}}$. Note that $p_{k}^{\mathrm{DA}, s}$ is the price for scheduling the quantity $e_{k}^{\mathrm{DA}, s}$, and $W_{k}^{\mathrm{DA}}$ is the amount of wind power production offered in the day-ahead market. The parameters $\beta$ and $\gamma_{k}$ control the shape of the supply curve. The value of $W_{k}^{\mathrm{DA}}$ is computed as

$$
W_{k}^{\mathrm{DA}}=\mathbb{E}\left[w_{k}\right] \bar{W},
$$

where $w_{k}$ is the normalized value $\left(w_{k} \in[0,1]\right)$ of wind power production, and $\bar{W}$ is the total installed wind capacity. The uncertain parameter $\gamma_{k}$ follows a Normal distribution, i.e.,

$$
\gamma_{k} \sim \mathcal{N}\left(\mu_{\gamma}, \sigma_{\gamma}^{2}\right)
$$

where $\mu_{\gamma}$ and $\sigma_{\gamma}^{2}$ are the mean value and variance of $\gamma_{k}$, respectively. In the balancing market, the supply curve at time interval $k$ is

$$
p_{k}^{\mathrm{BA}, s}= \begin{cases}\lambda^{0}, & \text { if } \Delta_{k}^{\mathrm{BA}, s} \leq 0 \\ \beta_{k}^{\mathrm{BA}} \Delta_{k}^{\mathrm{BA}, s}+\gamma_{k}^{\mathrm{BA}}\left(\Delta_{k}^{\mathrm{BA}, s}\right)^{2}+\lambda^{0}, & \text { otherwise }\end{cases}
$$

where $\Delta_{k}^{\mathrm{BA}, s}=e_{k}^{\mathrm{BA}, s}-e_{k}^{0}$. The variables $p_{k}^{\mathrm{BA}, s}$ and $e_{k}^{\mathrm{BA}, s}$ are the price and quantity of the balancing market supply curve, respectively. The term $e_{k}^{\mathrm{BA}, s}$ is obtained as the difference between $e_{k}^{\mathrm{DA}}$ and the imbalance generated by the stochastic generation, i.e.,

$$
e_{k}^{\mathrm{BA}, s}=e_{k}^{\mathrm{DA}}-\left(w_{k} \bar{W}-W_{k}^{\mathrm{DA}}\right) .
$$

Parameters $\beta_{k}^{\mathrm{BA}}$ and $e_{k}^{0}$ are evaluated by imposing that $p_{k}^{\mathrm{BA}, s}=$ $\lambda_{k}^{\mathrm{DA}}$ when $e_{k}^{\mathrm{BA}, s}=e_{k}^{\mathrm{DA}}$. This ensures that the day-ahead and the balancing market prices coincide when no balancing power is required.

\section{B. Scenario Generation}

We generate scenarios following a methodology presented in [21] and [22]. These papers propose a method for generating trajectories of a stochastic process when predictive distributions are available. The idea is to convert series of forecast errors into a multivariate Gaussian random variable and use a unique covariance matrix to describe its interdependence structure. This covariance matrix can be modeled through an exponential covariance function [22], where an exponential parameter $(\nu>1)$ controls the correlation between different lead times.

First, a set of scenarios $\left\{\gamma_{i k}: i \in I, k \in K\right\}$ is generated while fixing the exponential parameter $\nu$ to 5 . Then, a set of market price trajectories $\left\{\lambda_{i k}^{\mathrm{DA}}: i \in I, k \in K\right\}$ is generated, 
where the market-clearing price $\lambda_{i k}^{\mathrm{DA}}$ is obtained from the intersection between the demand and the supply curves at interval $k$ under scenario $i$.

For the balancing stage, we generate a set of wind power production trajectories $\left\{w_{j k}: j \in J, k \in K\right\}$ by fixing the exponential parameter $\nu$ to 7 as suggested in [22]. The probabilistic forecasts of wind power production are available in form of 19 quantiles (from 0.05 to 0.95 ). To fit the cumulative distribution function we follow the approach of [23]. Then, a set of market price scenarios $\left\{\lambda_{i j k}^{\mathrm{BA}}: i \in I, j \in J, k \in K\right\}$ is generated by clearing the balancing market model.

\section{Trading Problem Under a PAY-AS-BID Pricing SCHEME}

When trading in an electricity market, power producers can usually submit price-quantity offers. The quantity identifies the amount of energy they are willing to produce, and the price is the minimum price for which they are willing to produce that energy. Then, the offer is accepted only when the market price is higher than or equal to the offered one. Since the given producer is assumed to be price-taker, the market price in each future time interval is necessarily treated as an exogenous but uncertain parameter [4]-[5]. In this paper, the market price $\lambda$ is considered as a random variable following the density function $f^{\lambda}: \mathbb{R} \mapsto \mathbb{R}^{+}$. Given a price-quantity offer of a producer, denoted as $(p, q)$, the acceptance probability of the offer is

$$
\mathbb{P}[\lambda \geq p]=\int_{p}^{\infty} f^{\lambda}(l) \mathrm{d} l,
$$

where $l$ is an auxiliary integration variable. Under a pay-as-bid pricing scheme, the expected remuneration price $p^{*}$, providing that the producer's offer is being accepted, is computed as

$$
\mathbb{E}\left[p^{*} \mid \lambda \geq p\right]=p
$$

The expected return $\rho$ of the producer, following [18], is

$$
\mathbb{E}[\rho]=\mathbb{P}[\lambda \geq p] \mathbb{E}\left[p^{*} \mid \lambda \geq p\right] q .
$$

By replacing (7) and (8) in (9) we obtain

$$
\mathbb{E}[\rho]=q p \int_{p}^{\infty} f^{\lambda}(l) \mathrm{d} l .
$$

Notice that the expected return in (10) is non-linear. The offers in generation-side of real-world electricity market are generally non-decreasing step-wise functions, which can be modeled through a set of $B$ price-quantity offers $\left\{\left(p_{b}, q_{b}\right), b=1, . ., B\right\}$. The formulation in (10) is extended to the generic model (11) below including the multiple offer blocks, i.e.,

$$
\begin{array}{ll}
\underset{q_{b}, p_{b}}{\operatorname{Max}_{b}} & \sum_{b} \mathbb{E}\left[\rho_{b}\right]-\mathbb{E}\left[c_{b}\right] \\
\text { s.t. } & \mathbb{E}\left[\rho_{b}\right]=p_{b} q_{b} \int_{p_{b}}^{p_{b+1}} f^{\lambda}(l) \mathrm{d} l, \forall b \\
& \mathbb{E}\left[c_{b}\right]=h\left(q_{b}\right) \int_{p_{b}}^{p_{b+1}} f^{\lambda}(l) \mathrm{d} l, \forall b \\
& q_{b} \geq q_{b-1}, \forall b \\
& p_{b} \geq p_{b-1}, \forall b \\
& \underline{E} \leq q_{b} \leq \bar{E}, \forall b
\end{array}
$$

where $p_{B+1}=\infty$. The parameter $c_{b}$ is the operational cost for producing the quantity $q_{b}$, whose value is computed through the function $h(\cdot)$. Constraints (11b) and (11c) compute the expected return $\rho_{b}$ and the operational cost of the offering block $\left(p_{b}, q_{b}\right)$, respectively. Constraints (11d) and (11e) impose the non-decreasing condition of the offering curve. Finally, (11f) imposes the minimum and maximum production levels of the unit.

One of the main contributions of this work is to derive an alternative linear formulation to (11), which is non-linear. First, we discretize the continuous random variables. The uncertain market price can be represented using a set of possible scenarios $\left\{\lambda_{i}, i=1, \ldots, N\right\}$, where each price scenario $\lambda_{i}$ is associated with a probability $\pi_{i}$ such that $\sum_{i} \pi_{i}=1$. We consider each price scenario $\lambda_{i}$ as the potential offer price of the price-taker producer, and obtain the optimal quantity offer $q_{i}$ corresponding to each offer price $\lambda_{i}$. A collection of all price-quantity offers, i.e., $\left(\lambda_{i}, q_{i}\right)$, builds the offer curve of the producer, providing that the following conditions are enforced to ensure that the offer curve is non-decreasing [13]:

$$
\begin{aligned}
& q_{i} \geq q_{i^{\prime}} \text { if } \lambda_{i} \geq \lambda_{i^{\prime}}, \forall i, \forall i^{\prime}, \\
& q_{i}=q_{i^{\prime}} \text { if } \lambda_{i}=\lambda_{i^{\prime}}, \forall i, \forall i^{\prime},
\end{aligned}
$$

where $i$ and $i^{\prime}$ are indices of the market price scenarios. Note that this offer curve is now scenario-independent, i.e., it is adapted to all scenarios, though it is built based on scenario-dependent price-quantity offers. Under a uniform pricing scheme, the expected market return $\rho$ can be computed as

$$
\mathbb{E}[\rho]=\sum_{i} \pi_{i} \lambda_{i} q_{i},
$$

However, the market return formulation (13) needs to be changed under the pay-as-bid scheme, since each block offer is remunerated at its corresponding offer price. Therefore, we introduce variable $o_{i}$, which represents the additional quantity offered at price $\lambda_{i}$. Figure 1 illustrates an example offer curve with three offer blocks. In this curve, $\lambda_{1}, \lambda_{2}$ and $\lambda_{3}$ are not only the three price scenarios, but also they are price offers of the price-taker producer. For example, the producer offers its $q_{1} \mathrm{MWh}$ at price $\lambda_{1}$ (as the first offer block), and then the additional $o_{2}$ MWh (i.e., $q_{2}-q_{1}$ ) at price $\lambda_{2}$ (as the second offer block). The total quantity $q_{i}$ corresponding to price $\lambda_{i}$ can be computed as

$$
q_{i}=\sum_{i^{\prime}} M_{i i^{\prime}} o_{i^{\prime}},
$$

where the acceptance matrix $M$ is defined as

$$
M_{i i^{\prime}}= \begin{cases}1, & \text { if } \lambda_{i} \geq \lambda_{i^{\prime}} \\ 0, & \text { otherwise. }\end{cases}
$$

Matrix $M_{i i^{\prime}}$ indicates whether the offer block $\left(\lambda_{i^{\prime}}, o_{i^{\prime}}\right)$ is accepted in the market, providing that the market price realization is $\lambda_{i}$. The total expected return is computed as the sum of the expected returns for each offer block $\left(\lambda_{i^{\prime}}, o_{i^{\prime}}\right)$, denoted as $\rho_{i^{\prime}}$, i.e.,

$$
\mathbb{E}[\rho]=\sum_{i^{\prime}} \mathbb{E}\left[\rho_{i^{\prime}}\right] .
$$




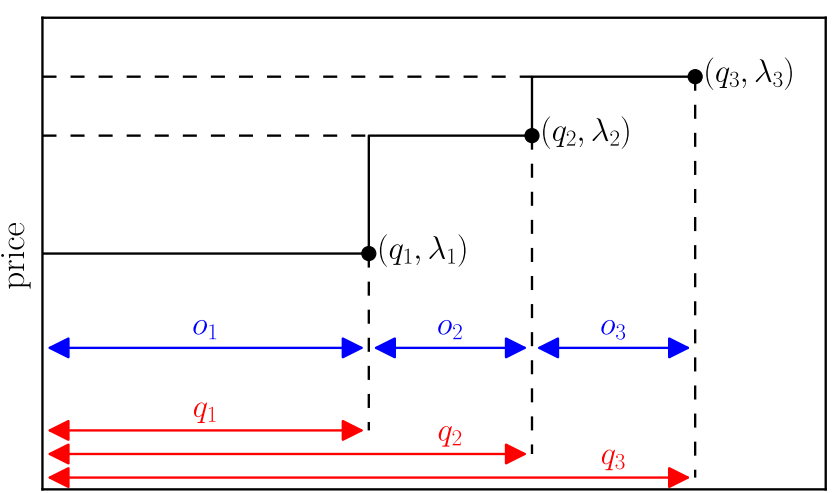

quantity

Fig. 1. An example offer curve with multiple blocks, which shows how market price scenarios are used as price offers, building an offer curve.

In line with (9), we compute the expectation of $\rho_{i^{\prime}}$ as

$$
\mathbb{E}\left[\rho_{i^{\prime}}\right]=\mathbb{P}\left[\lambda \geq \lambda_{i^{\prime}}\right] \mathbb{E}\left[p^{*} \mid \lambda \geq \lambda_{i^{\prime}}\right] o_{i^{\prime}},
$$

where the acceptance probability of each block offer is

$$
\mathbb{P}\left[\lambda \geq \lambda_{i^{\prime}}\right]=\sum_{i} M_{i i^{\prime}} \pi_{i},
$$

and the expected remuneration price is

$$
\mathbb{E}\left[p^{*} \mid \lambda \geq \lambda_{i^{\prime}}\right]=\lambda_{i^{\prime}} .
$$

Substituting (18) and (19) in (17) renders

$$
\mathbb{E}\left[\rho_{i^{\prime}}\right]=o_{i^{\prime}} \lambda_{i^{\prime}} \sum_{i} M_{i i^{\prime}} \pi_{i} .
$$

The total expected return can thus be computed as

$$
\mathbb{E}[\rho]=\sum_{i^{\prime}} o_{i^{\prime}} \lambda_{i^{\prime}} \sum_{i} M_{i i^{\prime}} \pi_{i} .
$$

Note that $(21)$ is linear. The expected profit $\mathbb{E}[\rho]$ can also be seen as $\sum_{i} \mathbb{E}\left[\rho_{i}\right]$, where $\rho_{i}$ is the return when scenario $i$ realizes. Therefore, we rewrite (21) as

$$
\mathbb{E}[\rho]=\sum_{i} \pi_{i} \rho_{i}=\sum_{i} \pi_{i} \sum_{i^{\prime}} M_{i i^{\prime}} \lambda_{i^{\prime}} o_{i^{\prime}} .
$$

Given the linear formulation in (22), we rewrite the generic nonlinear model (11) in a linear manner, i.e.,

$$
\begin{array}{ll}
\underset{\Theta}{\operatorname{Max}} & \sum_{i} \pi_{i}\left[\rho_{i}-c_{i}\right] \\
\text { s.t. } & q_{i}=\sum_{i^{\prime}} M_{i i^{\prime}} o_{i^{\prime}}, \forall i \\
& \rho_{i}=\sum_{i^{\prime}} M_{i i^{\prime}} \lambda_{i^{\prime}} o_{i^{\prime}}, \forall i \\
& c_{i}=h\left(q_{i}\right), \forall i \\
& \underline{E} \leq q_{i} \leq \bar{E}, \forall i
\end{array}
$$

where $\Theta=\left\{q_{i}, o_{i}, c_{i}, \rho_{i}, \forall i\right\}$. Constraints (23d) compute the production cost through the function $h(\cdot)$. It is worth mentioning that the non-decreasing conditions are not necessary in (23) since they are inherently included in the way we compute the accepted quantity $q_{i}$ in (23b). The performance of both models, i.e., (11) and (23), is analyzed and compared using a simple case study in Section V-A.

\section{Offering Strategy as a Two-Stage Stochastic OPTIMIZATION MODEL}

Given the linear offering strategy setup under a pay-asbid pricing scheme proposed in Section III, we derive the optimal offering curves of a conventional producer in the twosettlement electricity market described in Section II. In this model, we also consider the unit commitment constraints of that producer. At noon, the power producer has to submit its offering curves for the day-ahead market of the following day, based on the price scenarios for both day-ahead and balancing markets. In this two-stage setup, the producer maximizes its expected profit from both markets simultaneously, in the sense that it endogenously determines its future balancing actions while solving its offering problem in the day-ahead. Accordingly, we model the day-ahead production level in time interval $k$, i.e., $q_{k}^{\mathrm{DA}}$, as first-stage (here-and-now) decision, and the up and down production adjustments in the realtime stage, i.e., $q_{i k}^{\mathrm{UP}}$ and $q_{i k}^{\mathrm{DW}}$, as second-stage (wait-and-see) variables. Following the approach described in Section III for building the producer's offer curve, we now relax the day-ahead production variable to be scenario-dependent (i.e., $\left.q_{k}^{\mathrm{DA}} \rightarrow q_{i k}^{\mathrm{DA}}\right)$. Similarly, the real-time production adjustments $q_{i k}^{\mathrm{UP}}$ and $q_{i k}^{\mathrm{DW}}$ are made scenario-dependent (i.e., $q_{i k}^{\mathrm{UP}} \rightarrow q_{i j k}^{\mathrm{UP}}$, $\left.q_{i k}^{\mathrm{DW}} \rightarrow q_{i j k}^{\mathrm{DW}}\right)$ in order to build the producer's offer curve in the balancing stage. In addition, we add a detailed representation of the feasible operating region of the thermal unit. The optimization model that the power producer solves to decide the day-ahead market offers reads as follows

$$
\begin{array}{ll}
\operatorname{Max}_{\Xi} & \sum_{i j k} \pi_{i}^{\mathrm{DA}} \pi_{i j}^{\mathrm{BA}}\left[\lambda_{i k}^{\mathrm{DA}} q_{i k}^{\mathrm{DA}}+\rho_{i j k}^{\mathrm{UP}}-\rho_{i j k}^{\mathrm{DW}}-c_{i j k}\right] \\
\text { s.t. } & q_{i j k}^{\mathrm{A}}=q_{i k}^{\mathrm{DA}}+q_{i j k}^{\mathrm{UP}}-q_{i j k}^{\mathrm{DW}}, \forall i, \forall j, \forall k \\
& q_{i j k}^{\mathrm{UP}}=\sum_{j^{\prime}} M_{i j^{\prime} k}^{\mathrm{UP}} o_{i j^{\prime} k}^{\mathrm{UP}}, \forall i, \forall j, \forall k \\
& q_{i j k}^{\mathrm{DW}}=\sum_{j^{\prime}} M_{i j j^{\prime} k}^{\mathrm{DW}} o_{i j^{\prime} k}^{\mathrm{DW}}, \forall i, \forall j, \forall k \\
& \rho_{i j k}^{\mathrm{UP}}=\sum_{j^{\prime}} \lambda_{i j^{\prime} k}^{\mathrm{BA}} M_{i j j^{\prime} k}^{\mathrm{UP}} o_{i j^{\prime} k}^{\mathrm{UP}}, \forall i, \forall j, \forall k \\
& \rho_{i j k}^{\mathrm{DW}}=\sum_{j^{\prime}} \lambda_{i j^{\prime} k}^{\mathrm{BA}} M_{i j j^{\prime} k}^{\mathrm{DW}} o_{i j^{\prime} k}^{\mathrm{DW}}, \forall i, \forall j, \forall k \\
& q_{i k}^{\mathrm{DA}}, o_{i j k}^{\mathrm{UP}}, o_{i j k}^{\mathrm{DW}} \in \Pi, \forall i, \forall j, \forall k \\
& q_{i j k}^{\mathrm{A}}, u_{i j k}, y_{i j k}, z_{i j k} \in \Omega, \forall i, \forall j, \forall k \\
& c_{i j k}=\mathrm{g}\left(q_{i j k}^{\mathrm{A}}, u_{i j k}, y_{i j k}, z_{i j k}\right), \forall i, \forall j, \forall k \\
& q_{i k}^{\mathrm{DA}}, o_{i j k}^{\mathrm{UP}}, o_{i j k}^{\mathrm{DW}} \geq 0, \forall i, \forall j, \forall k \\
& u_{i j k}, y_{i j k}, z_{i j k} \in\{0,1\}, \forall i, \forall j, \forall k
\end{array}
$$

where

$$
\begin{aligned}
& \Xi=\left\{q_{i k}^{\mathrm{DA}}, o_{i j k}^{\mathrm{UP}}, o_{i j k}^{\mathrm{DW}}, q_{i j k}^{\mathrm{UP}}, q_{i j k}^{\mathrm{DW}}, \rho_{i j k}^{\mathrm{UP}}, \rho_{i j k}^{\mathrm{DW}}, q_{i j k}^{\mathrm{A}},\right. \\
& \left.u_{i j k}, y_{i j k}, z_{i j k}, x_{i j k s}, c_{i j k}: \forall i, \forall j, \forall j^{\prime}, \forall s, \forall k\right\} .
\end{aligned}
$$


The acceptance matrices $M_{j j^{\prime} k}^{\mathrm{UP}}$ and $M_{j j^{\prime} k}^{\mathrm{DW}}$ are defined as

$$
\begin{aligned}
& M_{i j j^{\prime} k}^{\mathrm{UP}}= \begin{cases}1, & \text { if } \lambda_{i j k}^{\mathrm{BA}} \geq \lambda_{i j^{\prime} k}^{\mathrm{BA}} \text { and } \lambda_{i j k}^{\mathrm{BA}}>\lambda_{i k}^{\mathrm{DA}} \\
0, & \text { otherwise, }\end{cases} \\
& M_{i j j^{\prime} k}^{\mathrm{DW}}= \begin{cases}1, & \text { if } \lambda_{i j k}^{\mathrm{BA}} \leq \lambda_{i j^{\prime} k}^{\mathrm{BA}} \text { and } \lambda_{i j k}^{\mathrm{BA}}<\lambda_{i k}^{\mathrm{DA}} \\
0, & \text { otherwise. }\end{cases}
\end{aligned}
$$

The objective function (24a) maximizes the expected profit of the producer from selling energy in both day-ahead and balancing markets. Constraints (24b) yield the total power production $q_{i j k}^{\mathrm{A}}$ when both day-ahead price scenario $i$ and balancing price scenario $j$ realize at time interval $k$. For the same scenario realization, constraints $(24 \mathrm{c})$ compute the level of up-regulation energy $q_{i j k}^{\mathrm{UP}}$ scheduled. Similarly, constraints (24d) obtain the level of down-regulation energy $q_{i j k}^{\mathrm{DW}}$. Constraints (24e) and (24f) give the expected revenues from selling regulation energy in the balancing market under a pay-as-bid pricing scheme. Constraints $(24 \mathrm{~g})$ include a set of constraints associated with the offer curves, which is represented later in Section IV-A. Constraints (24h) force the power producer to operate in its feasible operating region, which is provided in Section IV-B. Constraints (24i) compute the operational costs for given schedule, whose formulation is provided in Section IV-C.

Model (24) can be also used to compute the expected profit from a sequential offering approach. First, we solve the model considering the day-ahead scenarios only. To do that, we force the balancing variables to be null (i.e., $q_{i j k}^{\mathrm{UP}}=0$ and $q_{i j k}^{\mathrm{DW}}=$ $0 \forall i, j, k)$. The optimal solutions $\widetilde{q}_{i k}^{\mathrm{DA}}$ represent the optimal market offers when considering the day-ahead market only. Then, we solve again the model while imposing $q_{i k}^{\mathrm{DA}}=\widetilde{q}_{i k}^{\mathrm{DA}}$. The optimal solutions $\widetilde{q}_{i j k}^{\mathrm{UP} *}$ and $\widetilde{q}_{i j k}^{\mathrm{DW}}$ are the balancing market offers that maximize the expected profit, provided that the dayahead offers are $\widetilde{q}_{i k}^{\mathrm{DA}}$.

\section{A. Linear Expression of $\Pi$}

The offer curve constraints $(24 \mathrm{~g})$, denoted as $\Pi$, are

$$
\begin{aligned}
& q_{i k}^{\mathrm{DA}} \leq \bar{E}, \forall i, \forall k \\
& q_{i k}^{\mathrm{DA}} \leq q_{i^{\prime} k}^{\mathrm{DA}} \text { if } \lambda_{i k}^{\mathrm{DA}} \leq \lambda_{i^{\prime} k}^{\mathrm{DA}}, \forall i, \forall i^{\prime}, \forall k \\
& q_{i k}^{\mathrm{DA}}=q_{i^{\prime} k}^{\mathrm{DA}} \text { if } \lambda_{i k}^{\mathrm{DA}}=\lambda_{i^{\prime} k}^{\mathrm{DA}}, \forall i, \forall i^{\prime}, \forall k \\
& o_{i j^{\prime} k}^{\mathrm{UP}}=0 \text { if } \lambda_{i j^{\prime} k}^{\mathrm{BA}} \leq \lambda_{i k}^{\mathrm{DA}}, \forall i, \forall j^{\prime}, \forall k \\
& o_{i j^{\prime} k}^{\mathrm{DW}}=0 \text { if } \lambda_{i j^{\prime} k}^{\mathrm{BA}} \geq \lambda_{i k}^{\mathrm{DA}}, \forall i, \forall j^{\prime}, \forall k
\end{aligned}
$$

Constraints (26a) restrict the day-ahead production quantity of the producer to its capacity. Constraints (26b) and (26c) enforce the non-decreasing and non-anticipativity conditions of the producer's offer curve in the day-ahead, respectively. These two conditions are required for offer curves to be submitted to markets settled under a uniform pricing scheme. Constraints (26d) and (26e) impose that no balancing energy is contracted when it is not required by the system.

\section{B. Linear Expression of $\Omega$}

Constraints $(24 \mathrm{~h})$ represent the feasible operating region of the producer and can be replaced by

$$
\begin{aligned}
& q_{i j k}^{\mathrm{A}} \leq u_{i j k} \bar{E}, \forall i, \forall j, \forall k \\
& q_{i j k}^{\mathrm{A}} \geq u_{i j k} \underline{E}, \forall i, \forall j, \forall k \\
& q_{i j k}^{\mathrm{A}}-q_{i j(k-1)}^{\mathrm{A}} \leq R^{\mathrm{UP}}, \forall i, \forall j, \forall k \\
& q_{i j(k-1)}^{\mathrm{A}}-q_{i j k}^{\mathrm{A}} \leq R^{\mathrm{DW}}, \forall i, \forall j, \forall k \\
& u_{i j k}-u_{i j(k-1)} \leq y_{i j k}, \forall i, \forall j, \forall k \\
& u_{i j(k-1)}-u_{i j k} \leq z_{i j k}, \forall i, \forall j, \forall k
\end{aligned}
$$

Constraints (27a) and (27b) impose that the total power production lies between its minimum and maximum production levels. Constraints (27c) and (27d) enforce ramp-up and rampdown limits [3]. Constraints (27e) and (27f) determine the start-up status $y_{i j k}$ and the shut-down status $z_{i j k}$. Notice that constraints $(27 \mathrm{c})-(27 \mathrm{f})$ require the initial production level and commitment status $\left(q_{0}^{\mathrm{A}}, u_{0}\right)$.

\section{Linear Expression of Operational Cost}

Constraints (24i) define the operational cost of the thermal unit and can be replaced by

$$
\begin{aligned}
& c_{i j k}=C_{0} u_{i j k}+\sum_{s} C_{s} x_{i j k s}+ \\
& +C^{\mathrm{UP}} y_{i j k}+C^{\mathrm{DW}} z_{i j k}, \forall i, \forall j, \forall k \\
& 0 \leq x_{i j k s} \leq E_{s}, \forall i, \forall j, \forall k, \forall s \\
& \underline{E} u_{i j k}+\sum_{s} x_{i j k s}=q_{i j k}^{\mathrm{A}}, \forall i, \forall j, \forall k .
\end{aligned}
$$

Constraints (28a) compute the operational cost of the unit including its start-up and shut-down costs [4]. Constraints (28b) and (28c) are auxiliary constraints representing a linear formulation of the quadratic cost function through a piecewise approximation [3], where $x_{i j k s}$ is the amount of energy produced by the generation block $s$.

\section{Case Study}

\section{A. LP Trading Model Verification}

In this section we test and compare the proposed LP model (23) and the existing NLP model (11) using a simple test case, considering a single time period. The market price $\lambda$ follows a Normal distribution, i.e.

$$
\lambda \sim \mathcal{N}(50,5) .
$$

The production unit has a capacity $\bar{E}$ of $60 \mathrm{MW}$ and a minimum production level $\underline{E}$ of $0 \mathrm{MW}$. The quadratic cost function $h(\cdot)$ is approximated with a two-step piecewise linear cost function, i.e.,

$$
\begin{aligned}
& h(q)=C_{1} x_{1}+C_{2} x_{2}, \\
& q=x_{1}+x_{2}, \\
& 0 \leq x_{1} \leq E_{1}, \\
& 0 \leq x_{2} \leq E_{2},
\end{aligned}
$$


where $E_{1}$ and $E_{2}$ are $30 \mathrm{MW}$, while $C_{1}=€ 35 / \mathrm{MWh}$ and $C_{2}$ $=€ 47 / \mathrm{MWh}$.

The input scenarios for the LP model (23) are selected following the scenario reduction technique in [24]. We generate 1000 scenarios from (29) and then keep the 20 most representative ones. The LP model is implemented using GUROBI [25] in Python, and it is solved in around 0.001s. The optimal solution obtained is given in Table I, where the expected profit $\mathbb{E}[\rho]$ is re-computed considering the continuous distribution of $\lambda$ in (29). The NLP continuous model (11) is solved using COBYLA [26] algorithm in PYTHON in around 0.115 s. Its optimal solution is also reported in Table I. The two models show similar optimal solutions, while the gap in the expected profit due to the discretization procedure is lower than $0.07 \%$. The LP model is solved around 115 times faster than the NLP one. It is worth mentioning that the computational time of the NLP model increases to around $3.2 \mathrm{~s}$ when the integrals in (11) are numerically computed, instead of using the cumulative distribution function of (29). However, the main advantage of the LP formulation is to be more suitable to be merged with the unit commitment constraints of the thermal unit. Therefore, a comparison between a MILP and MINLP offering model would be more appropriate to test the advantages of our LP formulation. Nevertheless, the solution of a MINLP problem is out of the scope of this paper.

This test case shows that the LP formulation, when provided with an accurate sampling of input scenarios, brings an optimal solution close to the continuous NLP alternative. In the following section, we extend the LP model by including the unit commitment constraints of the thermal unit in a multitime period and multi-stage stochastic optimization problem.

\section{B. Day-ahead Offering Model Test Case}

We test the two-stage stochastic optimization model (24) on a realistic case study. We generate market price scenarios according to the methodology presented in Section II. The input parameters are shown in Tables II and III. First, we generate 300 scenarios for $\lambda_{i k}^{\mathrm{DA}}$ and we select the 20 most representative ones. Then, for each scenario $\lambda_{i k}^{\mathrm{DA}}$, we generate 300 scenarios of $\lambda_{i j k}^{\mathrm{BA}}$ and keep the 20 most representative ones. This procedure results in a scenario tree with 400 branches. We repeat this sampling procedure for different values of $\bar{W}$, i.e. 10,20 and $30 \mathrm{GW}$. Figure 2 shows the 20 scenarios of $\lambda_{i k}^{\mathrm{DA}}$ (in blue) and the 20 scenarios of $\lambda_{i j k}^{\mathrm{BA}}$ (in green) for a given realization of $\lambda_{k}^{\mathrm{DA}}$ (in red), when $\frac{i j k}{W}$ $=20 \mathrm{GW}$. We consider a thermal unit with a capacity of $\bar{E}=120 \mathrm{MW}$ and a minimum production level of $\underline{E}=40$

TABLE I

THE PRODUCER'S OPTIMAL MARKET OFFERS OBTAINED FROM THE LP AND NLP MODELS

\begin{tabular}{ccccc}
\hline & & $\begin{array}{c}q_{b} \\
(\mathrm{MWh})\end{array}$ & $\begin{array}{c}\lambda_{b} \\
(€ / \mathrm{MWh})\end{array}$ & $\begin{array}{c}\mathbb{E}[\rho] \\
(€)\end{array}$ \\
\hline \multirow{2}{*}{ NLP } & $b=1$ & 30 & 46.6 & 313.4 \\
& $b=2$ & 60 & 51.7 & \\
LP & $b=1$ & 30 & 46.4 & 313.2 \\
& $b=2$ & 60 & 51.8 & \\
\hline
\end{tabular}

TABLE II

PARAMETERS OF THE MARKET PRICE GENERATION MODEL

\begin{tabular}{ccccc}
\hline $\begin{array}{c}\delta \\
\left(€ / \mathrm{MWh}^{2}\right)\end{array}$ & $\begin{array}{c}\beta \\
\left(€ / \mathrm{MWh}^{2}\right)\end{array}$ & $\begin{array}{c}\mu_{\gamma} \\
\left(€ / \mathrm{MWh}^{3}\right)\end{array}$ & $\begin{array}{c}\sigma_{\gamma}^{2} \\
\left(€ / \mathrm{MWh}^{3}\right)\end{array}$ & $\begin{array}{c}\lambda^{0} \\
(€ / \mathrm{MWh})\end{array}$ \\
\hline$-6.67 \times 10^{-3}$ & $1 \times 10^{-4}$ & $2 \times 10^{-8}$ & $3 \times 10^{-9}$ & -20 \\
\hline
\end{tabular}

TABLE III

VALUES OF PARAMETER $\alpha_{k}$

\begin{tabular}{ccccccccc}
\hline$k$ & 1 & 2 & 3 & 4 & 5 & 6 & 7 & 8 \\
$\alpha_{k}(€ / \mathrm{MWh})$ & 322 & 312 & 315 & 317 & 340 & 349 & 353 & 369 \\
\hline$k$ & 9 & 10 & 11 & 12 & 13 & 14 & 15 & 16 \\
$\alpha_{k}(€ / \mathrm{MWh})$ & 394 & 424 & 444 & 445 & 440 & 429 & 437 & 458 \\
\hline$k$ & 17 & 18 & 19 & 20 & 21 & 22 & 23 & 24 \\
$\alpha_{k}(€ / \mathrm{MWh})$ & 446 & 423 & 408 & 383 & 373 & 346 & 331 & 332 \\
\hline
\end{tabular}
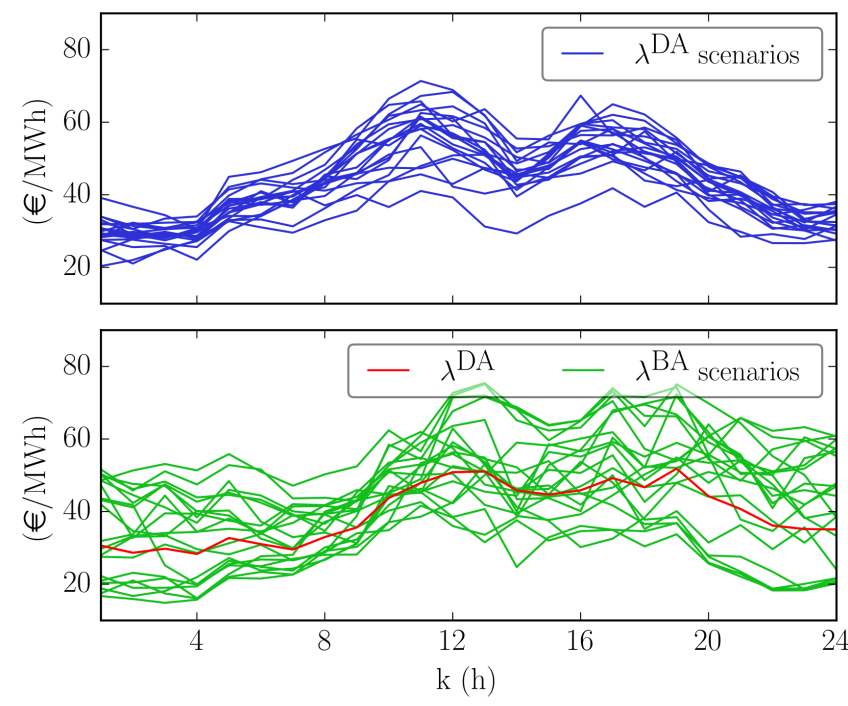

Fig. 2. Day-ahead and balancing market price scenarios.

MW. Ramping limits are $40 \mathrm{MW}$ for both $R^{\mathrm{UP}}$ and $R^{\mathrm{DW}}$. The quadratic cost function is approximated by a piecewise linear function of four generation blocks of equal size, i.e. $E_{s}=20$ MW $\forall s$. Table IV shows the marginal cost $C_{s}$ of each block, the cost $C_{0}$, the start-up cost $C^{\mathrm{UP}}$ and the shut-down cost $C^{\mathrm{DW}}$.

The optimization model is implemented using GUROBI in PyThON environment. We compare the two-stage cooptimization model with a sequential offering approach. Indeed, by modeling balancing market variables as recourse decisions at the day-ahead stage, we co-optimize the offering strategy for the two markets.

As an example, Table $\mathrm{V}$ reports the optimal value of the dayahead production variable $q_{i k}^{\mathrm{DA}}$ in time interval $k=7$ obtained from both the co-optimized and sequential approaches. Note that $\lambda_{i 7}^{\mathrm{DA}}, i=1, \ldots, 20$, is the set of the day-ahead price scenarios, and each member of this set is viewed as a potential price offer. For the co-optimized approach, the results given 
TABLE IV

PARAMETERS OF THE COST FUNCTION

\begin{tabular}{ccccccc}
\hline $\begin{array}{c}C_{0} \\
(€)\end{array}$ & $\begin{array}{c}C_{s_{1}} \\
(€ / \mathrm{MWh})\end{array}$ & $\begin{array}{c}C_{s_{2}} \\
(€ / \mathrm{MWh})\end{array}$ & $\begin{array}{c}C_{S_{3}} \\
(€ / \mathrm{MWh})\end{array}$ & $\begin{array}{c}C_{s_{4}} \\
(€ / \mathrm{MWh})\end{array}$ & $\begin{array}{c}C^{\mathrm{UP}} \\
(€)\end{array}$ & $\begin{array}{c}C^{\mathrm{DW}} \\
(€)\end{array}$ \\
\hline 2860 & 23.5 & 31.5 & 45.6 & 72.3 & 800 & 100 \\
\hline
\end{tabular}

in Table V can be summarized as given in (31) below:

$$
q_{7}^{\mathrm{DA}}= \begin{cases}0, & \text { if } \lambda_{7}^{\mathrm{DA}}<39.7 \\ 40, & \text { if } 39.7 \leq \lambda_{7}^{\mathrm{DA}}<42.0 \\ 80, & \text { if } 42.0 \leq \lambda_{7}^{\mathrm{DA}}<51.7 \\ 120, & \text { if } \lambda_{7}^{\mathrm{DA}} \geq 51.7\end{cases}
$$

where $q_{7}^{\mathrm{DA}}$ is expressed in $\mathrm{MWh}$ and $\lambda_{7}^{\mathrm{DA}}$ in $€ / \mathrm{MWh}$. According to (31), a scenario-independent offer curve in dayahead can be built using three price-quantity offer points, i.e., $(€ 39.7 / \mathrm{MWh}, 40 \mathrm{MW}),(€ 42.0 / \mathrm{MWh}, 80 \mathrm{MW})$ and $(€ 51.7 / \mathrm{MWh}, 120 \mathrm{MW})$. A graphic representation of this curve is provided in Figure 3a, blue curve. Similarly, the dayahead offer curve at time interval $k=7$ for the sequential approach can be obtained (red curve). Note that in both approaches, the producer is not willing to produce if $\lambda_{7}^{\mathrm{DA}} \leq$ 39.7 while desires to operate at its full capacity if $\lambda_{7}^{\mathrm{DA}} \geq$ 51.7. However, when $42.0 \leq \lambda_{7}^{\mathrm{DA}} \leq 48.2$, the co-optimized approach suggests to produce $80 \mathrm{MW}$, while the sequential approach does $40 \mathrm{MW}$ only. In addition, Figure 3b shows the offering curve of the producer in the balancing market at time interval $k=7$, provided that the realization of dayahead price $\lambda_{7}^{\mathrm{DA}}$ is $€ 44.1 / \mathrm{MWh}$. Based on the co-optimized approach, the producer is scheduled to produce $80 \mathrm{MW}$ and then to reduce its production level in the balancing stage to $40 \mathrm{MW}$ if $\lambda_{7}^{\mathrm{BA}} \leq 35.6$, or to increase it to $120 \mathrm{MW}$ in case $\lambda_{7}^{\mathrm{BA}} \geq 55.7$. Unlike the co-optimized approach, the sequential one schedules the producer at $40 \mathrm{MW}$ in the dayahead market, and then provides the up-regulation service only in the balancing stage. For instance, its production increases by $40 \mathrm{MW}$ if $\lambda_{7}^{\mathrm{BA}} \geq 56.0$ while that increase is even more ( 80 $\mathrm{MW}$ ) in case $\lambda_{7}^{\mathrm{BA}} \geq 58.7$. The expected profits obtained from these two approaches under different conditions are shown in Table VI. In a case in which $\bar{W}=10 \mathrm{GW}$, the expected profit loss in the sequential approach is around $2 \%$. The power producer gains a lower expected profit in the day-ahead market while earning more in the balancing stage, such that its total expected profit (including both stages) increases as well. This behavior is more observable in the cases with a higher value of installed wind capacity. For instance, the loss of profit is $22 \%$ and $91 \%$ in cases in which $\bar{W}$ is equal to 20 and $30 \mathrm{GW}$, respectively. The last case ( $\bar{W}=30 \mathrm{GW})$ gives more insight: in the sequential approach, the producer does not participate in the day-ahead market, and earns a low profit in the balancing stage only. In contrast, the producer gains a significant money in the co-optimized approach, though it loses money in the day-ahead stage. In fact, it takes such a loosing position in day-ahead market to be able to produce profitable regulation services in the balancing stage.
TABLE V

OPTIMAL VALUES OF $q_{i 7}^{\text {DA }}$ FOR THE SEQUENTIAL AND CO-OPTIMIZED APPROACHES

\begin{tabular}{|c|c|c|c|c|c|c|c|}
\hline \multirow[t]{2}{*}{$i$} & \multirow[t]{2}{*}{$\begin{array}{c}\lambda_{i 7}^{\mathrm{DA}} \\
(€ / \mathrm{MWh})\end{array}$} & \multicolumn{2}{|c|}{$\begin{array}{c}q_{i 7}^{\mathrm{DA}} \\
(\mathrm{MWh})\end{array}$} & \multirow[t]{2}{*}{$i$} & \multirow[t]{2}{*}{$\begin{array}{c}\lambda_{i 7}^{\mathrm{DA}} \\
(€ / \mathrm{MWh})\end{array}$} & \multicolumn{2}{|c|}{$\begin{array}{c}q_{i 7}^{\mathrm{DA}} \\
(\mathrm{MWh})\end{array}$} \\
\hline & & co-opt & seq & & & co-opt & seq \\
\hline 1 & 44.1 & 80 & 40 & 11 & 36.3 & 0 & 0 \\
\hline 2 & 33.0 & 0 & 0 & 12 & 44.4 & 80 & 40 \\
\hline 3 & 45.1 & 80 & 40 & 13 & 44.6 & 80 & 40 \\
\hline 4 & 45.6 & 80 & 40 & 14 & 43.4 & 80 & 40 \\
\hline 5 & 39.7 & 40 & 40 & 15 & 52.7 & 120 & 120 \\
\hline 6 & 37.1 & 0 & 0 & 16 & 44.7 & 80 & 40 \\
\hline 7 & 48.2 & 80 & 80 & 17 & 42.0 & 80 & 40 \\
\hline 8 & 44.9 & 80 & 40 & 18 & 41.1 & 40 & 40 \\
\hline 9 & 51.7 & 120 & 120 & 19 & 44.3 & 80 & 40 \\
\hline 10 & 43.0 & 80 & 40 & 20 & 46.9 & 80 & 40 \\
\hline
\end{tabular}

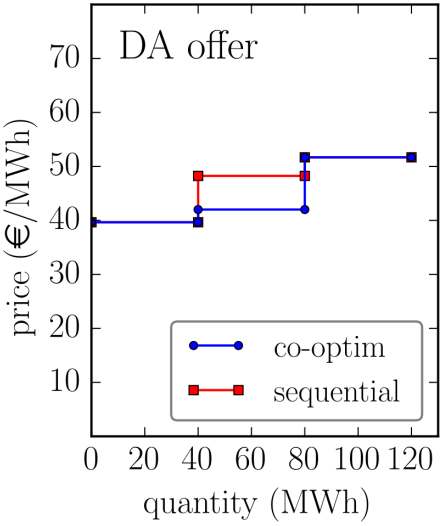

(a)

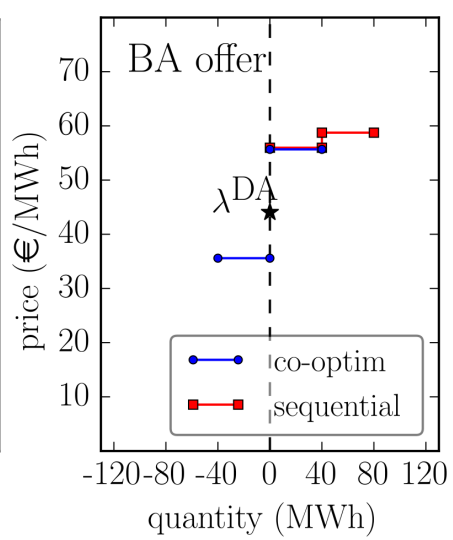

(b)
Fig. 3. The producer's optimal offer curve in (a) day-ahead market, (b) balancing market at time interval $k=7(\bar{W}=20 \mathrm{GW})$.

\section{CONClusions AND FurTher Works}

This paper presents a novel method for deriving optimal offering curves of a price-taker conventional producer in an electricity market under a pay-as-bid pricing scheme. The importance of this study is that several European balancing markets in the balancing stage are settled under a pay-as-bid pricing scheme. The main contribution of this paper is that we develop an LP approach. In contrast, the existing tools in the literature are mainly non-linear and less suitable to be merged to the MILP feasibility region of a thermal unit. Then, we extend our proposed approach to a two-stage market setup including day-ahead and balancing stages. In this setup, being consistent with the structure of several European electricity markets, the day-ahead market is cleared based on a uniform

TABLE VI

EXPECTED PROFIT OF THE PRODUCER

\begin{tabular}{ccccc}
\hline $\begin{array}{c}\bar{W} \\
(\mathrm{GW})\end{array}$ & Model & $\begin{array}{c}\text { Profit in DA } \\
\left(10^{3} €\right)\end{array}$ & $\begin{array}{c}\text { Profit in BA } \\
\left(10^{3} €\right)\end{array}$ & $\begin{array}{c}\text { Total } \\
\left(10^{3} €\right)\end{array}$ \\
\hline \multirow{2}{*}{10} & co-optimized & 16.82 & 3.25 & 20.09 \\
& sequential & 18.08 & 1.59 & 19.68 \\
20 & co-optimized & 4.45 & 8.27 & 12.27 \\
& sequential & 7.68 & 2.27 & 9.95 \\
\multirow{2}{*}{30} & co-optimized & -8.45 & 18.44 & 9.99 \\
& sequential & 0.00 & 0.87 & 0.87 \\
\hline
\end{tabular}


pricing scheme, while a pay-as-bid scheme is used in the balancing stage. To make our setup more realistic, we include the unit commitment constraints of the thermal units to our proposed setup, resulting in a MILP model.

We first test our proposed single-stage LP model against the existing non-linear models. Our LP formulation successes in well approximating the non-linear one. Then, we compare our proposed two-stage MILP model against a sequential offering model, which does not consider the balancing stage while offering in the day-ahead market. Our proposed approach shows a better performance in terms of expected profit achieved.

In future research it is of interest to test the proposed model using real market price data. Besides, intra-day markets could be included in the offering model. These additional trading floors may bring more business opportunities to the producer. Moreover, the proposed approach could be extended to derive the optimal offering strategy of different technologies, such as energy storage systems. For such facilities, the participation in the balancing market may be significantly important for exploiting their flexibility.

\section{ACKNOWLEDGMENT}

The authors would like to thank Lesia Mitridati (DTU) for discussion and feedback on previous versions of the work, and Stefanos Delikaraoglou (DTU) and Christos Ordoudis (DTU) for their comments on the final manuscript. The authors would also like to thank the anonymous reviewers for their valuable comments and suggestions which have improved the quality of this paper.

\section{REFERENCES}

[1] F. Sensfuss, M. Ragwitz, and M. Genoese, "The merit-order effect: A detailed analysis of the price effect of renewable electricity generation on spot market prices in Germany," Energy Policy, vol. 36, no. 8, pp. 3086-3094, 2008.

[2] S. Clò, A. Cataldi, and P. Zoppoli, "The merit-order effect in the Italian power market: The impact of solar and wind generation on national wholesale electricity prices," Energy Policy, vol. 77, pp. 79-88, 2015.

[3] J. M. Arroyo and A. J. Conejo, "Optimal response of a thermal unit to an electricity spot market," IEEE Trans. Power Syst., vol. 15, no. 3, pp. 1098-1104, 2000

[4] _ , "Modeling of start-up and shut-down power trajectories of thermal units," IEEE Trans. Power Syst., vol. 19, no. 3, pp. 1562-1568, 2004.

[5] A. J. Conejo, F. J. Nogales, J. M. Arroyo, and R. García-Bertrand, "Riskconstrained self-scheduling of a thermal power producer," IEEE Trans. Power Syst., vol. 19, no. 3, pp. 1569-1574, 2004.

[6] R. A. Jabr, "Robust self-scheduling under price uncertainty using conditional value-at-risk," IEEE Trans. Power Syst., vol. 20, no. 4, pp. 1852-1858, 2005.

[7] A. J. Conejo, F. J. Nogales, and J. M. Arroyo, "Price-taker bidding strategy under price uncertainty," IEEE Trans. Power Syst., vol. 17, no. 4, pp. 1081-1088, 2002.

[8] E. Ni, P. B. Luh, and S. Rourke, "Optimal integrated generation bidding and scheduling with risk management under a deregulated power market," IEEE Trans. Power Syst., vol. 19, no. 1, pp. 600-609, 2004.

[9] M. Maenhoudt and G. Deconinck, "Strategic offering to maximize dayahead profit by hedging against an infeasible market clearing result," IEEE Trans. Power Syst., vol. 29, no. 2, pp. 854-862, 2014.

[10] A. Baillo, M. Ventosa, M. Rivier, and A. Ramos, "Optimal offering strategies for generation companies operating in electricity spot markets," IEEE Trans. Power Syst., vol. 19, no. 2, pp. 745-753, 2004.

[11] V. P. Gountis and A. G. Bakirtzis, "Bidding strategies for electricity producers in a competitive electricity marketplace," IEEE Trans. Power Syst., vol. 19, no. 1, pp. 356-365, 2004.
[12] A. G. Bakirtzis, N. P. Ziogos, A. C. Tellidou, and G. A. Bakirtzis, "Electricity producer offering strategies in day-ahead energy market with step-wise offers," IEEE Trans. Power Syst., vol. 22, no. 4, pp. 18041818, 2007.

[13] A. J. Conejo, M. Carrión, and J. M. Morales, Decision Making Under Uncertainty in Electricity Markets. Springer, 2010.

[14] Q. Wang, C. Zhang, Y. Ding, G. Xydis, J. Wang, and J. Østergaard, "Review of real-time electricity markets for integrating distributed energy resources and demand response," Applied Energy, vol. 138, pp. 695-706, 2015.

[15] Y. Ren and F. D. Galiana, "Pay-as-bid versus marginal pricing - part I: Strategic generator offers," IEEE Trans. Power Syst., vol. 19, no. 4, pp. 1771-1776, 2004

[16] — - "Pay-as-bid versus marginal pricing - part II: Market behavior under strategic generator offers," IEEE Trans. Power Syst., vol. 19, no. 4, pp. 1777-1783, 2004

[17] D. J. Swider and C. Weber, "Bidding under price uncertainty in multi-unit pay-as-bid procurement auctions for power systems reserve," European Journal of Operational Research, vol. 181, no. 3, pp. 12971308, 2007.

[18] D. J. Swider, "Simultaneous bidding in day-ahead auctions for spot energy and power systems reserve," International Journal of Electrical Power \& Energy Systems, vol. 29, no. 6, pp. 470-479, 2007.

[19] J. Khorasani and H. R. Mashhadi, "Bidding analysis in joint energy and spinning reserve markets based on pay-as-bid pricing," IET Generation, Transmission \& Distribution, vol. 6, no. 1, pp. 79-87, 2012.

[20] J. Sadeh, H. R. Mashhadi, and M. A. Latifi, "A risk-based approach for bidding strategy in an electricity pay-as-bid auction," European Transactions on Electrical Power, vol. 19, no. 1, pp. 39-55, 2009.

[21] P. Pinson, H. Madsen, H. A. Nielsen, G. Papaefthymiou, and B. Klöckl, "From probabilistic forecasts to statistical scenarios of short-term wind power production," Wind Energy, vol. 12, no. 1, pp. 51-62, 2009.

[22] P. Pinson and R. Girard, "Evaluating the quality of scenarios of shortterm wind power generation," Applied Energy, vol. 96, pp. 12-20, 2012.

[23] P. H. D. Andersen, "Optimal trading strategies for a wind-storage power system under market conditions," Master's thesis, Technical University of Denmark, DTU, DK-2800 Kgs. Lyngby, Denmark, 2009.

[24] N. Growe-Kuska, H. Heitsch, and W. Romisch, "Scenario reduction and scenario tree construction for power management problems," in IEEE Power Tech Conference Proceedings, 2003.

[25] Gurobi Optimization, "Gurobi optimizer reference manual," 2016. [Online]. Available: http://www.gurobi.com

[26] E. Jones, T. Oliphant, P. Peterson et al., "SciPy: Open source scientific tools for Python," 2001. [Online]. Available: http://www.scipy.org

Nicolò Mazzi received the B.Sc. degree in 2011 and the M.Sc. degree in 2014, both from the University of Padova, Padova, Italy, in energy engineering. He is currently pursuing the $\mathrm{Ph} . \mathrm{D}$. degree at the Department of Industrial Engineering of University of Padova. His research interests include energy systems, electricity markets, stochastic optimization and decomposition techniques.

Jalal Kazempour (M14) is an assistant professor at the Department of Electrical Engineering, Technical University of Denmark, Kgs. Lyngby, Denmark. He received his Ph.D. degree in electrical engineering from University of Castilla-La Mancha, Ciudad Real, Spain, in 2013. His research interests include power systems, electricity markets, optimization, and its applications to energy systems.

Pierre Pinson (M11-SM13) received the M.Sc. degree in applied mathematics from the National Institute for Applied Sciences (INSA Toulouse, France) and the Ph.D. degree in energetics from Ecole des Mines de Paris (France). He is a Professor at the Technical University of Denmark (DTU), Centre for Electric Power and Energy, Department of Electrical Engineering, also heading a group focusing on Energy Analytics \& Markets. His research interests include among others forecasting, uncertainty estimation, optimization under uncertainty, decision sciences, and renewable energies. Prof. Pinson acts as an Editor for the International Journal of Forecasting, and for Wind Energy. 